\title{
Estudo comparativo das características fluidodinâmicas de próteses valvulares biológicas de pericárdio bovino de perfil alto e baixo
}

Aron J. P. ANDRADE*. José F. BISCEGLI*, Denys E. NICOLOSI*, Hernán C. GÓMEZ*, J. Eduardo M. R. SOUSA*

RBCCV 44205-98

ANDRADE, A. J. P.; BISCEGLI, J. F.; NICOLOSI, D. E.; GÓMEZ, H. C.; SOUSA, J. E. M. R. - Estudo comparativo das características fluidodinâmicas de próteses valvulares biológicas de pericárdio bovino de perfil alto e baixo. Rev. Bras. Cir. Cardiovasc., 4(3): 231-236, 1989.

RESUMO: O comportamento fluidodinâmico e a vida útil média de uma prótese valvular cardiaca são características muito importantes na escolha do modelo de prótese a ser implantada em um determinado paciente. Por esse motivo, foi realizado um estudo comparativo entre as válvulas biológicas de perfil alto e baixo, para se verificarem possíveis alteraçōes na vida média, gradiente de pressão e refluxo, causadas pela diminuição do perfil dessas próteses. Com o auxilio de um duplicador de pulso, foram analisadas cinco próteses de perfil alto e cinco de perfil baixo, todas tamanho 29, fabricadas no Instituto Dante Pazzanese de Cardiologia (IDPC). Mantendo-se a freqüência de batimentos em $100 \mathrm{bpm}$ e a diferença de pressão em $1000 \mathrm{mmHg}$, verificou-se que a vida útil média da válvula de perfil baixo permaneceu equivalente à de perfil alto. Em outro equipamento, denominado simulador cardiaco, as próteses foram submetidas a condiçōes semelhantes às condiçōes fisiológicas de funcionamento para uma frequiência cardiaca de $75 \mathrm{bpm}$. Os resultados mostraram que o volume de retorno necessário para fechamento (refluxo), nas válvulas de perfil baixo, é menor do que nas de perfil alto. Isso traz um melhor aproveitamento do efeito de bombeamento do coração. As perdas de carga das válvulas foram comparadas através dos gradientes máximos de pressão visibilizados com a sobreposição dos sinais de pressão. Observou-se que as válvulas de perfil baixo oferecem menos resistência ao fluxo; assim, quando são implantadas, possibilitam uma passagem mais livre e desobstruída do sangue.

DESCRITORES: próteses valvulares cardíacas, testes; próteses valvulares cardiacas, biológicas.

\section{INTRODUÇÃO}

A primeira prótese valvular cardiaca foi implantada por Hunfnagel, em 1953 (MAZUNDAR \& THALASSOUDIS $^{12}$ ), fato que, na época, foi considerado um grande sucesso. Isso impulsionou diversos pesquisadores de todo o mundo a estudarem e aperfeiçoarem novos modelos de válvulas cardiacas. As áreas de Engenharia e
Medicina dos grandes centros de pesquisa, trabalhando em conjunto, já desenvolveram mais de 60 outros tipos de próteses (comprovadamente implantadas), umas com mais sucesso que outras. Entretanto, através da análise dos inúmeros artigos publicados a esse respeito ${ }^{3,6,10 .}$ $14,15,19$, pode-se concluir que ainda não foi desenvolvida nenhuma válvula cardiaca artificial que seja considerada uma substituta ideal da valva natural. Isso se deve à

Trabalho realizado no Instituto Dante Pazzanese de Cardiologia. São Paulo, SP, Brasil.

Recebido para publicaçáo em 25 de outubro de 1989.

- Do Instituto Dante Pazzanese de Cardiologia.

Endereço para separatas: Aron J. P. Andrade. Av. Dr. Dante Pazzanese, 500. Centro Técnico de Pesquisas e Experimentos. 04512 Săo Paulo, SP, Brasil. 
ANDRADE, A. J. P.; BISCEGLI, J. F.; NICOLOSI, D. E.; GOMMEZ, H. C.; SOUSA, J. E. M. R. - Estudo comparativo das características fluidodinâmicas de próteses valvulares biológicas de pericárdio bovino de perfil alto e baixo. Rev. Bras. Cir. Cardiovasc., 4(3): 231-236, 1989.

necessidade de um tratamento do paciente com drogas anticoagulantes quando são implantadas próteses mecânicas, ou devido à vida útil das próteses biológicas ser reduzida ${ }^{11}$ (aproximadamente 10 anos).

O comportamento fluidodinâmico de uma prótese é uma característica muito importante para o seu bom desempenho, tornando uma prótese mais aconselhável que a outra de outro modelo, ou com funcionamento diferente. Assim, as características fluidodinâmicas de uma prótese são uma das diversas variáveis que influenciam na escolha do modelo de prótese a ser implantado em determinado paciente.

Um estudo municioso das características fluidodinâmicas das próteses faz-se necessário, para a obtenção de resultados numéricos que auxiliem no desenvolvimento de novos modelos, assim como aperfeiçoamento e compreensão dos já existentes. Para isso, são utilizados sistemas simuladores cardiacos, também conhecidos como analisadores de válvulas cardiacas, que as submetem a fluxos pulsáteis, simulando as press̄ōes fisiológicas das válvulas mitral e aórtica, levando-se em consideração a configuração anatômica de ventrículo esquerdo, átrio esquerdo e aorta.

Existe um outro tipo de equipamento diferente do simulador cardiaco, denominado acelerador de pulso, que, por sua vez, possibilita a avaliaçāo da resistência mecânica à fadiga ou ao desgaste e, ainda, a vida média das próteses.

O objetivo deste trabalho é realizar um estudo comparativo entre as características das válvulas biológicas de pericárdio bovino de perfil alto e as de perfil baixo, para se verificar se houve alteraçōes de desempenho com a diminuição do perfil das próteses. Para tanto, é necessário e suficiente comparar as vidas úteis médias, os refluxos e os gradientes de pressão das próteses ${ }^{18}$.

\section{MATERIAL E MÉTODOS}

\section{Acelerador de Pulso}

O princípio básico de funcionamento do acelerador de pulso consiste na elevação das pressōes e da velocidade de batimento das próteses cardiacas ${ }^{1,2}$. TrabaIhando em condiçōes forçadas, as próteses se desgastam mais rapidamente, chegando, muitas vezes, a se romper ${ }^{11}$. Assim, com a utilização de fatores de multiplicaçāo relativos às condiçōes de pressāo e velocidade impostas às válvulas pelo acelerador, consegue-se analisar, qualitativamente, as variáveis desejadas: fadiga, desgaste e vida média. Com o auxilio de uma lâmpada estroboscópica com freqüência regulável, pode-se observar com clareza os movimentos de abertura e o fechamento das válvulas.

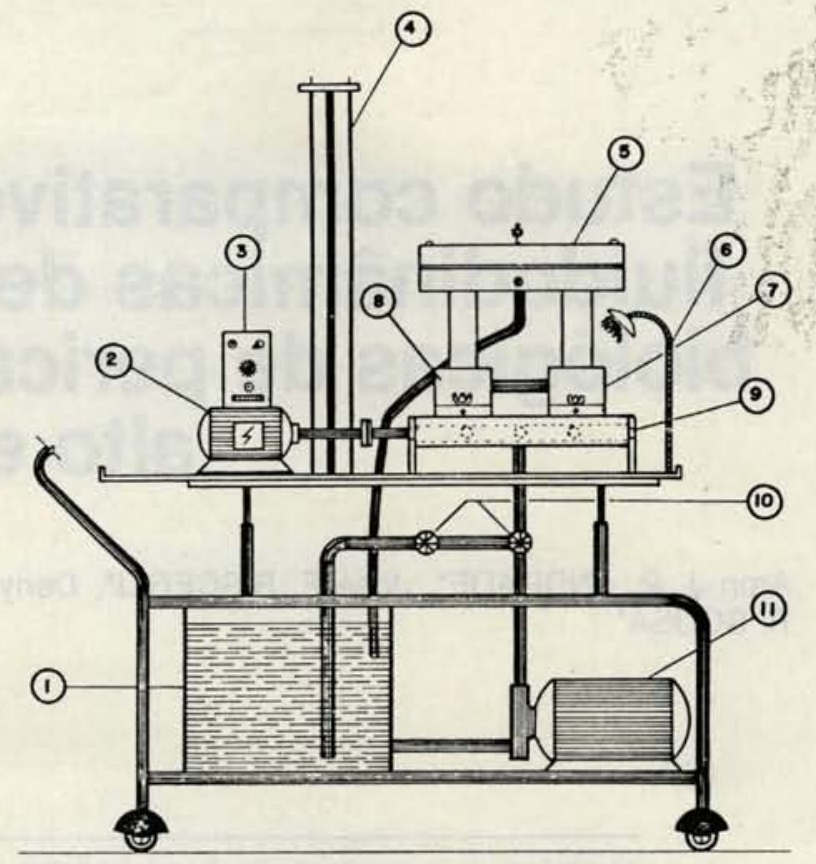

Fig. 1 - Acelerador de pulso: 1 = reservatório; 2 = motor CC com regulagem de velocidade; 3 = circuito do motor; $4=$ coluna de mercúrio das pressōes pré e pós-câmara; 5 = câmara de equilibrio de pressão; 6 = lâmpada estroboscópica; 7 = câmara I; 8 = câmara II; 9 = eixo de reversão da câmara de teste $; 10$ = registro de vazăo e ladrão; 11 = motor-bomba d’água.

Foi desenvolvido, no Instituto Dante Pazzanese de Cardiologia (IDPC), um modelo de acelerador de pulso que atende, perfeitamente, às necessidades no estudo das resistências mecânicas das próteses, como mostra a Figura 1.

Nesse equipamento, foram testadas cinco válvulas de pericárdio de perfil baixo e cinco de perfil alto, todas de tamanho 29, colocadas duas a duas no acelerador, de modo a ficarem lado a lado, uma de perfil alto e outra de perfil baixo. Dessa forma, foram feitos cinco ensaios, onde se encontravam funcionando uma válvula de perfil alto e uma de perfil baixo, ao mesmo tempo; assim, conseguiram-se, exatamente, as mesmas condições de funcionamento para ambos os modelos, em cada um dos ensaios. As condições mantidas durante os ensaios foram:

- As freqüências de batimento foram mantidas em 1000 bpm, reguladas através da rotação do motor (2) da Figura 1 (1000 rpm). Esse motor movimenta um eixo (9) com rebaixos estrategicamente desenvolvidos para reverter as pressōes nas câmaras de testes (7 e 8).

- A diferença de pressão existente antes e após cada válvula, no período em que ela se encontrava fechada, era de $1000 \mathrm{mmHg}$, visibilizadas através de colunas de mercúrio (4). 
ANDRADE, A. J. P.; BISCEGLI, J. F.; NICOLOSI, D. E.; GOMEZ, H. C.; SOUSA, J. E. M. R. - Estudo comparativo das características fluidodinâmicas de próteses valvulares biológicas de pericárdio bovino de perfil alto e baixo. Rev. Bras. Cir. Cardiovasc. 4(3): 231-236, 1989.

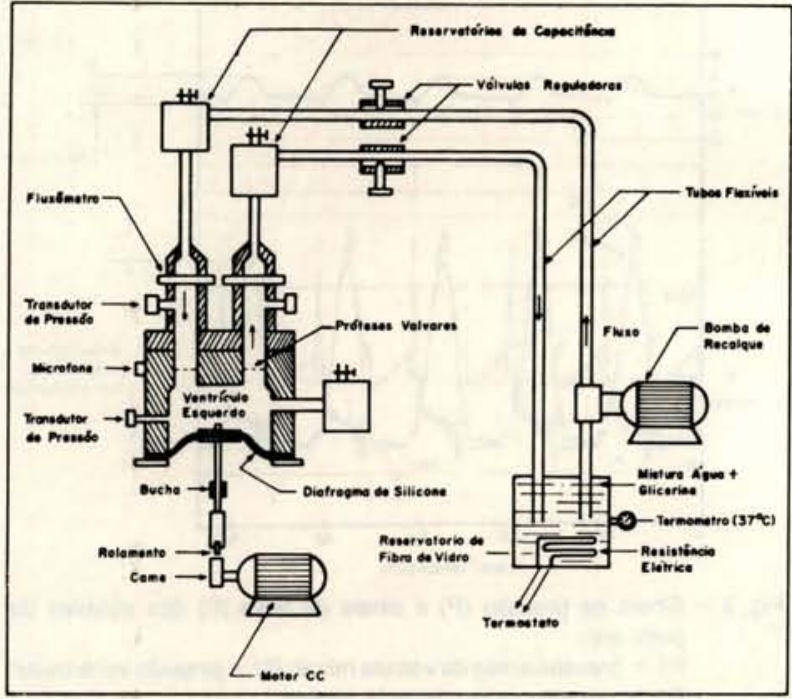

Fig. 2 - Simulador cardiaco.

- As pressões eram ajustadas através das válvulas (10) de regulagem colocadas após a bomba de recalque (11).

- Cada válvula foi mantida nessas condições críticas de funcionamento, até o rompimento total ou parcial de pelo menos um dos três folhetos, ou quebra de pelo menos uma das três hastes que compōem a estrutura plástica deste tipo de válvula.

\section{Simulador Cardíaco}

O simulador cardíaco é um aparelho que deve proporcionar às válvulas artificiais um fluxo pulsátil com propriedades semelhantes às fisiológicas ${ }^{13}$, para que se possa testar as características fluidodinâmicas das próteses valvulares em funcionamento. Assim, utiliza-se uma mistura de $60 \%$ de água e $40 \%$ de glicerina, com viscosidade dinâmica ( 3 e 4 centipoise) e densidade $\left(1,07 \mathrm{~g} / \mathrm{cm}^{3}\right)$ semelhante às do sangue ${ }^{1}$.

A Figura 2 mostra, em detalhes, as partes que compõem o simulador cardiaco, como: sistema mecânico, câmaras de ensaio e sistema hidráulico.

A mistura é proveniente de um reservatório com volume suficiente para assegurar um fornecimento adequado ao sistema. O calor é fornecido ao fluido por uma resistência elétrica que liga e desliga automaticamente, por intermédio de um termostato ajustado em $37^{\circ} \mathrm{C}$, com tolerância de $2^{\circ} \mathrm{C}$. Há homogeneidade de temperatura dentro do tanque, devido ao constante movimento do líqüido.

O fluxo é obtido através do movimento de um diafragma de silicone conectado a câmaras de tubos acrilicos, que representam o sistema circulatório.
O mecanismo que movimenta o diafragma de silicone (simulando o batimento cardíaco) é constituído de um motor elétrico de corrente contínua, com circuito retificador e controlador de velocidade. No motor é conectado um came ${ }^{5}$, para possibilitar tempos diferentes de sístole e diástole, reproduzindo o que ocorre no coração. Por exemplo: para uma freqüência de $75 \mathrm{bpm}$, o período do ciclo é de 0,8 segundos, onde o tempo de sístole corresponde a 0,35 segundos e o de diástole a 0,45 segundos.

No simulador cardíaco, foram realizados dois ensaios:

O primeiro com duas válvulas de pericárdio bovino de perfil alto, tamanho 29, construídas no IDPC, uma colocada em posição mitral e outra em posição aórtica, na câmara que simula o ventrículo esquerdo.

O segundo ensaio foi realizado com duas válvulas de perfil baixo, também tamanho 29 , construídas no IDPC, nas mesmas condiçōes do primeiro ensaio.

As válvulas em teste foram fixadas na câmara de acrílico, levando-se em conta o posicionamento das mesmas no ventrículo esquerdo do coração humano ${ }^{4}$. Tal geometria tende a aumentar a semelhança das condiçōes de funcionamento da prótese no simulador, comparando-se com as condiçōes reais de funcionamento.

As pressões ou diferenças de pressões, existentes antes e após as válvulas (mitral e aórtica), foram obtidas através de transdutores fisiológicos de pressão, elementos utilizados para converter uma grandeza hemodinâmica em elétrica com muita rapidez e alta sensibilidade a pequenas variaçōes de pressāo.

As vazōes foram obtidas por cabeçotes medidores de fluxo por processo eletromagnético (adicionados 3\% de solução fisiológica, para aumentar a condutividade elétrica da mistura), instalados após a válvula aórtica, na câmara que simula a aorta. Em seguida, os dados de vazão foram manipulados e devidamente registrados em forma de sinais gráficos.

Para registrar e analisar os sinais de pressão e fluxo, foi utilizado um polígrafo da Hewlett-Packard, ou seja, um monitor HP 1308A, um sistema de controle HP 5692A, um registrador de papel HP7785A e também um fluxômetro Statham SP 22011.

Em complementação a esses dados de pressão e vazão, coletados e armazenados para posterior análise, as válvulas foram também estudadas em pleno funcionamento através do acrílico, visibilizando-se suas dinâmicas de abertura e fechamento para diversos niveis de fluxo, bem como a visibilização (com o auxílio de partículas em suspensão) ${ }^{7,8,16}$ do comportamento do fluido, onde se pode perceber a existência, ou não, de zonas de turbulência próximas às válvulas ${ }^{3}$, áreas de recirculação e linhas de velocidade e de pressão ${ }^{12}$. 
ANDRADE, A. J. P.; BISCEGLI, J. F.; NICOLOSI, D. E.; GOMEZ, H. C.; SOUSA, J. E. M. R. - Estudo comparativo das características fluidodinâmicas de próteses valvulares biológicas de pericárdio bovino de perfil alto e baixo. Rev. Bras. Cir. Cardiovasc., $4(3): 231-236,1989$.

\section{Resultados Obtidos no Acelerador de Pulso}

Os resultados dos testes comparativos da resistência mecânica realizados em 10 próteses, obtidos no acelerador de pulso são apresentados na Tabela 1.

Comparando-se os tempos totais de batimentos das válvulas de perfil alto e de perfil baixo, verificou-se que não houve nenhuma diferença significativa nas vidas úteis médias de cada modelo. Através deste estudo, não é possivel determinar, quantitativamente, a vida útil de uma prótese; apenas pode-se realizar um estudo comparativo, pois este estudo envolve diversas variáveis e fatores que são difíceis de controlar. Um desses fatores é o processo de deteriorização do pericárdio, uma vez que se encontra, durante os testes, em meio nāo estéril.

\section{Resultados Obtidos no Simulador Cardiaco}

Os resultados típicos no simulador cardíaco são mostrados nas Figuras 3 e 4.

\section{TABELA 1}

RESULTADOS COMPARATIVOS OBTIDOS NO ACELERADOR DE PULSO

\begin{tabular}{|c|c|c|c|}
\hline $\begin{array}{l}\text { Nido } \\
\text { ensaio }\end{array}$ & $\begin{array}{l}\text { Tipo de } \\
\text { válvula }\end{array}$ & $\begin{array}{c}\text { Tempo total de } \\
\text { batimentos (horas) }\end{array}$ & $\begin{array}{c}\text { Danos à } \\
\text { válvula }\end{array}$ \\
\hline \multirow{2}{*}{1} & Perfil alto & 36 & $\begin{array}{l}\text { Quebra da haste } \\
\text { do suporte }\end{array}$ \\
\hline & Perfil baixo & 33 & $\begin{array}{l}\text { Rotura do } \\
\text { pericárdio }\end{array}$ \\
\hline \multirow{2}{*}{2} & Perfil alto & 40 & $\begin{array}{l}\text { Rotura do } \\
\text { pericárdio }\end{array}$ \\
\hline & Perfil baixo & 45 & $\begin{array}{l}\text { Rotura do } \\
\text { pericárdio }\end{array}$ \\
\hline \multirow{2}{*}{3} & Perfil alto & 40 & $\begin{array}{l}\text { Rotura do } \\
\text { pericárdio }\end{array}$ \\
\hline & Perfil baixo & 40 & $\begin{array}{l}\text { Rotura do } \\
\text { pericárdio }\end{array}$ \\
\hline \multirow[b]{2}{*}{4} & Perfil alto & 38 & $\begin{array}{l}\text { Quebra da haste } \\
\text { do suporte }\end{array}$ \\
\hline & Perfil baixo & 43 & $\begin{array}{l}\text { Rotura do } \\
\text { pericárdio }\end{array}$ \\
\hline \multirow{2}{*}{5} & Perfil alto & 45 & $\begin{array}{l}\text { Rotura do } \\
\text { pericárdio }\end{array}$ \\
\hline & Perfil baixo & 35 & $\begin{array}{l}\text { Quebra da haste } \\
\text { do suporte }\end{array}$ \\
\hline
\end{tabular}

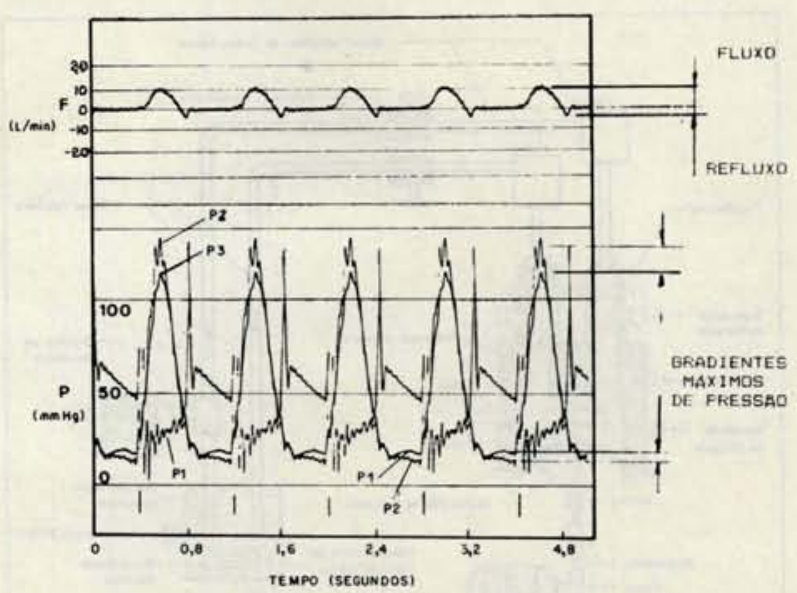

Fig. 3 - Sinais de pressão $(P)$ e sinais de fluxo $(F)$ das válvulas de perfil alto.

P1 = pressâo antes da válvula mitral; P2 = pressão ventricular; P3 = pressão após a válvula aórtica.

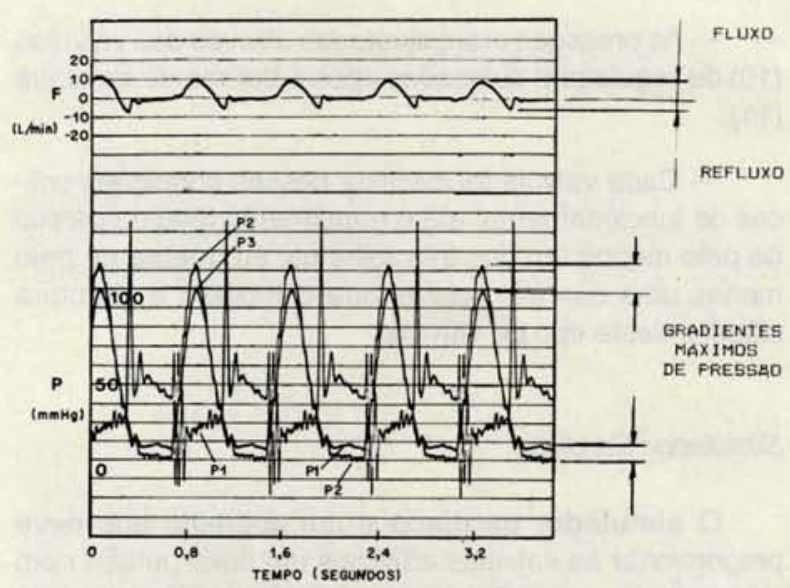

Fig. 4 - Sinais de pressăo $(P)$ e sinais de fluxo $(F)$ das válvulas de perfil baixo.

P1 = pressão antes da válvula mitral; P2 = pressão ventricular; P3 = pressão após a válvula aórtica

Fixando as diversas variáveis de funcionamento do simulador cardíaco, pode-se realizar um estudo comparativo entre os diferentes modelos de próteses valvulares.

A análise visual dos sinais de pressão e fluxo das válvulas comparadas no simulador cardíaco mostrouse suficiente para a realização de estudo qualitativo de algumas características fluidodinâmicas ${ }^{17}$; comparandose os sinais de fluxo das válvulas, verificou-se que o refluxo da válvula de perfil alto é maior que o refluxo da de perfil baixo, pois, fazendo-se a relaçăo entre o fluxo positivo (fluxo) e o fluxo negativo (refluxo) de cada válvula, notou-se que a altura do fluxo negativo da válvula de perfil alto é, aproximadamente, metade da altura do positivo. Isso se dá devido ao fato de que as válvulas 
ANDRADE, A. J. P.; BISCEGLI, J. F.; NICOLOSI, D. E.; GOMEZ, H. C.; SOUSA, J. E. M. R. - Estudo comparativo das características fluidodinâmicas de próteses valvulares biológicas de pericárdio bovino de perfil alto e baixo. Rev. Bras. Cir. Cardiovasc., $4(3): 231-236,1989$.

de perfil alto possuem folhetos maiores, necessitando, assim, de maior volume de líqüido retornando, para se fecharem. O refluxo deve ser o menor possivel, pois prejudica a circulaçāo sangüinea, que deve ocorrer apenas em um único sentido. $O$ refluxo foi estudado apenas na posição aórtica, pois apresenta maior volume de refluxo do que na posição mitral (devido à queda rápida da pressão ventricular esquerda, no início da diástole).

Durante o tempo em que a válvula em teste permanece aberta, nota-se uma diferença entre as pressōes antes e após a válvula. O que se quer comparar é essa queda de pressão causada pela resistência ao fluxo ou perda de carga de válvulas do mesmo tamanho, porém de modelos diferentes ${ }^{8}{ }^{18}$. No simulador, tal queda de pressāo é mais sensível na válvula instalada na posição aórtica, pois ela trabalha em niveis maiores de pressão e fluxo, e o tempo de sístole é menor que o de diástole ${ }^{4}$.

Analisando os sinais de pressão, verificou-se que, no intervalo de tempo em que a válvula aórtica se encontra aberta, a pressão ventricular (P2) é maior do que a pressão após a válvula (P3). No pico de pressão, existe uma diferença máxima entre as pressōes P2 e P3, que é denominada "Gradiente Máximo de Pressão na Válvula Aórtica". No intervalo de tempo em que a válvula mitral permanece aberta, a pressão antes da válvula mitral (P1) é maior do que a pressão ventricular (P2) e a diferença máxima entre P1 e P2 é denominada "Gradiente Máximo de Pressão na Válvula Mitral". Comparando os gradientes máximo de pressāo das válvulas de perfil alto (Figura 3 ) e das de perfil baixo (Figura 4), verifica-se que existe muito pouca diferença entre eles, mas os gradientes mostrados na Figura 3 são um pouco maiores que os gradientes mostrados na Figura 4. Isso indica que as válvulas de perfil baixo possuem menor gradiente de pressão, pois suas hastes são mais curtas e seus folhetos se abrem mais que nas válvulas de perfil alto, o que possibilita passagem mais fácil do fluido pela válvula e, no caso de implante, o coraçāo irá trabalhar mais livremente, com pressão ventricular menor.

\section{CONCLUSÃO}

Através dos testes comparativos realizados com válvulas de perfil alto e perfil baixo, todas tamanho 29 , construidas no IDPC, pode-se concluir que a diminuição do perfil das válvulas biológicas não prejudica seu funcionamento. Quanto à vida útil destas próteses, não houve nenhuma alteração significativa que tornasse proibitiva a diminuição do perfil da válvula. Por outro lado, o fato do refluxo da válvula de perfil baixo ser menor é muito importante e indica que este modelo deve ser preferivel, uma vez que traz menos prejuízo à circulação sangüínea, isto é, quando uma válvula está implantada em qualquer uma das posições possíveis, o volume de sangue deslocado em sentido contrário ao da circulaçāo é menor (esse volume é necessário para fechar os folhetos da prótese, porém deve ser o menor possível).

As perdas de carga das válvulas foram comparadas através dos gradientes máximos de pressão visibilizados com a sobreposição dos sinais de pressão obtidos nos ensaios dos dois modelos de válvulas. Verificou-se que a prótese de perfil baixo apresentou gradientes de pressão menores, oferecendo, conseqüentemente, menor resistência ao fluxo, isso devido ao fato de seus flhetos abrirem mais do que as válvulas de perfil alto. Isso mostra que a diminuição do perfil da válvula provocou melhora no seu comportamento fluidodinâmico, pois possibilita uma passagem mais livre ou desobstruída do sangue, o que proporciona melhor aproveitamento da energia de bombeamento do coração, transformada em energia cinética do sangue.

ANDRADE, A. J. P.; BISCEGLI, J. F.; NICOLOSI, D. E.; GOMEZ, H. C.; SOUSA, J. E. M. R. - Comparative study of fluid dynamic characteristics in high and low biological prosthetic heart valves from bovine pericardium. Rev. Bras. Cir. Cardiovasc., 4(3): 231-236, 1989.

ABSTRACT: The fluid dynamic performance and lifetime of a prosthetic heart valve are one of the most important characteristics to determine the choice of what prosthesis must be applied to a patient. Because of this, two different biological prosthetic heart valves profiles (one high profile and other low profile) were studied, to verify possible alterations of working life, pressure gradient and reverse flux debt to this profiles difference. With a Pulse Duplication, ten valves sixe 29 were analysed, manufactured by Instituto Dante Pazzanese de Cardiologia, being five low profile and five high profile. With a working frequency of $1000 \mathrm{ppm}$ (pulses per minute) and a differential pressure of $1000 \mathrm{mmHg}$, we verified the same working life in both cases. With a Cardiac Simulator, the prosthesis was submitted to similar physiologic human cardiac conditions, with an equivalent $75 \mathrm{ppm}$ heart pulse. The results show that the reverse flux volume necessary to close the low profile valve is lower than with the high profile valve. The loss head of these valves were compared though the maximum pressure gradient of them. It was noticed that the low profile , biological prosthetic valves are lower flux resistant than the high profile valves, being then more indicated to its implant function.

DESCRIPTORS: heart valves prostheses, tests; heart valves prostheses, biologic. 
ANDRADE, A. J. P.; BISCEGLI, J. F.; NICOLOSI, D. E.; GOMEZ, H. C.; SOUSA, J. E. M. R. - Estudo comparativo das características fluidodinâmicas de próteses valvulares biológicas de pericárdio bovino de perfil alto e baixo. Rev. Bras. Cir. Cardiovasc., 4(3): 231-236, 1989.

\section{REFERÊNCIAS BIBLIOGRÁFICAS}

1 ANDRADE, A. J. P. - Simulador cardiaco para teste de próteses valvares. Campinas, 1987. [Tese. Mestrado. Universidade Estadual de Campinas].

2 ANDRADE, A. J. P.; DISNER, A. F.; BISCEGLI, J. F.; GOMEZ, H. C.; ARRUDA, A. C. F. - Simulador cardiaco para teste de próteses valvares. Rev. Eng. Bioméd., 6: 480-487, 1989.

3 BELHOUSE, B. J. - Fluid mechanics of a model mitral valve and left ventricle. Cardiovasc. Res., 6:199-210, 1972.

4 BELHOUSE, B. J. \& WILLIAMS, W. - From left atrium to left ventricle. INSERM, 111: 411-426, 1982.

5 BENEDETTI, F.; MATTARA, M.; MILAUSKI, J. A.; PATANE, A. M. - Ensayo comparativo de prótesis valvulares en un duplicador de flujo pulsante. Rev. Arg. Cardiol., 51: $71-78,1983$.

6 CORNHILL, J. F. - An aortic-left ventricular pulse duplicator used in testing prosthetic aortic heart valves. J. Thorac. Cardiovasc. Surg., 73: 550-558, 1977.

7 DAVEY, T. B.; KAUFMAN, B.; SMELOFF, E. A. - Pulsatile flow studies of prosthetic heart valves. J. Thorac. Cardiovasc. Surg., 51: 264-267, 1966.

8 DUFF, W. R. \& FOX, R. W. - Prosthetic cardiac valves, and in vitro study. J. Thorac. Cardiovasc. Surg., 63: 131-141, 1972.

9 ENGELMAN, M. S.; MOKOWITZ, S. E.; BORNAN, J. B. - Computer simulation: a diagnostic method in comparative studies of valve prostheses. J. Thorac. Cardiovasc. Surg., 79: 402-412, 1980.

10 FIGUERA-AYMERICH, F. D. \& CASTILLO-OLIVARES, J. L. C. - A manual pulse duplicador to test heart valves before implantation. J. Thorac. Cardiovasc. Surg., 66: 25-28, 1973.
11 GABBAY, S.; BORTOLOTTI, U.; WASSERMAN, F.; FACTOR, S. M. - Haemodynamics and durability of mitral bioprostheses: an in vitro study. Am. Heart J., 5 (Supl. D): 65-71, 1984.

12 MAZUNDAR, J. \& THALASSOUDIS, K. - A mathematical model for the study of flow through disc-type prosthetic heart valves. Med. Biol. Eng. Comput., 21: $400-409$, 1983.

13 SILVA, M. R. - Fisiologia da circulação. Sāo Paulo, Edart, 1973.

14 SMELOFF, E. A.; HUNTLEY, A. C.; DAVEY, T. B.; KAUFMAN, B.; GERBODE, F. - Comparative study of prosthetic heart valves. J. Thorac. Cardiovasc. Surg., 52 : 841-848, 1966.

15 STARKEY, W. L.: SIRAK, H. D.; COLLINS, J. A.; HAGAN, B. T. - The design and development of a cardiac simulator for the evaluation of heart-valve prosthesis. J. Thorac. Cardiovasc. Surg., 46: 207-211, 1963.

16 ST. JUDE MEDICAL Inc. - All-pyrolytic carbon heart valve. Apresentado à Annual Meeting Association for the Advancement of Medical Instrumentation, 13. Washington DC, 1978. (Anais, p. 1-30).

17 SUOBANK, D. W.; YOGANATHAN, A. P.; HARRISON, E. C.; CORCORAN, W. H. - A quantitative method for the in vitro study of sounds produced by prosthetic aortic heart valves: part I, II and III. Med. Biol. Eng. Comput., 22: 32-54, 1984.

18 WALKER, D. K.; SCOTTEN, L. N.; MODI, V. J.; BROWLEE, R. T. - In vitro assessment of mitral valve prostheses. J. Thorac. Cardiovasc. Surg., 79:680-688, 1980.

19 WILLSHAW, P.; BIAGETTI, M.; PICHEL, R. H. - In vitro study of the closing characteristics of prosthetic mitral valves. Congresso Brasileiro de Engenharia Biomédica. 9. Universidade Estadual de Campinas, 1985 (Anais, p. 29). 